\title{
On Bialostocki's conjecture for zero-sum sequences
}

by

\author{
Song Guo (Huaian) and ZHI-WeI Sun (Nanjing)
}

1. Introduction. A finite sequence $S$ of terms from an (additive) abelian group is said to be a zero-sum sequence if the sum of the terms of $S$ is zero. In 1961 P. Erdős, A. Ginzburg and A. Ziv [3] proved that any sequence of $2 n-1$ terms from an abelian group of order $n$ contains an $n$-term zerosum subsequence. This celebrated EGZ theorem is an important result in combinatorial number theory and it has many different generalizations [5-8] including Sun's recent extension involving covering systems.

The following theorem is called the weighted EGZ theorem. It was conjectured by Y. Caro [2] and proved by D. J. Grynkiewicz [4].

Theorem 1.1 (Weighted EGZ Theorem). Let $n$ be a positive integer and let $w_{1}, \ldots, w_{n} \in \mathbb{Z}_{n}=\mathbb{Z} / n \mathbb{Z}$ with $\sum_{k=1}^{n} w_{k}=0$. If $a_{1}, \ldots, a_{2 n-1}$ is a sequence of elements from $\mathbb{Z}_{n}$, then $\sum_{k=1}^{n} w_{k} a_{j_{k}}=0$ for some distinct $j_{1}, \ldots, j_{n} \in\{1, \ldots, 2 n-1\}$.

Recently Bialostocki raised the following challenging conjecture.

Conjecture 1.1 (Bialostocki [1, Conjecture 14]). Let $n$ be a positive even integer. Suppose that $a_{1}, \ldots, a_{n}$ and $w_{1}, \ldots, w_{n}$ are zero-sum sequences with terms from $\mathbb{Z}_{n}$. Then there exists a permutation $\sigma \in S_{n}$ such that $\sum_{k=1}^{n} w_{k} a_{\sigma(k)}=0$, where $S_{n}$ denotes the symmetric group of all permutations on $\{1, \ldots, n\}$.

The conjecture has been verified for $n=2,4,6,8$. It fails for $n=$ $3,5,7, \ldots$. For example, $\left\{a_{1}, a_{2}, a_{3}\right\}=\left\{w_{1}, w_{2}, w_{3}\right\}=\mathbb{Z}_{3}$ gives a counterexample for $n=3$.

In this paper we mainly establish the following result.

TheOREM 1.2. Let $n$ be a positive even integer, and let $a_{1}, \ldots, a_{n} \in \mathbb{Z}$ with $\sum_{k=1}^{n} a_{k} \equiv 0(\bmod n)$. Then there exists a permutation $\sigma \in S_{n}$ such

2010 Mathematics Subject Classification: Primary 11B75; Secondary 05A05, 20D60.

Key words and phrases: zero-sum sequence, Bialostocki's conjecture, Erdős-Ginzburg-Ziv theorem. 
that $\sum_{k=1}^{n} k a_{\sigma(k)} \equiv 0(\bmod n / 2)$. Consequently, if $w_{1}, \ldots, w_{n} \in \mathbb{Z}$ form an arithmetic progression with even common difference, then $\sum_{k=1}^{n} w_{k} a_{\sigma(k)} \equiv 0$ $(\bmod n)$ for some $\sigma \in S_{n}$.

We are going to present two lemmas in the next section and then give our proof of Theorem 1.2 in Section 3.

\section{Two lemmas}

Lemma 2.1. Let $n=m q$ with $m, q \in \mathbb{Z}^{+}=\{1,2,3, \ldots\}$ and $m \geq 2$. Let $d \in \mathbb{Z}^{+}$be a divisor of $q$, and let $a_{1}, \ldots, a_{n} \in \mathbb{Z}$. Then there is a partition $I_{1}, \ldots, I_{m}$ of $[1, n]=\{1, \ldots, n\}$ such that for each $s=1, \ldots, m$ we have $\left|I_{s}\right|=q$ and

$$
d\left|\sum_{i \in I_{s}} a_{i} \Rightarrow\right|\left\{a_{i} \bmod d: i \in I_{s}\right\} \mid=1 .
$$

Proof. By induction on $m$, it suffices to show that there exists an $I \subseteq$ $[1, n]$ with $|I|=q$ such that for each $J \in\{I,[1, n] \backslash I\}$ we have $\mid\left\{a_{j} \bmod d\right.$ : $j \in J\} \mid=1$ or $\sum_{j \in J} a_{j} \not \equiv 0(\bmod d)$. To achieve this we distinguish three cases.

CASE 1: $\left|\left\{a_{i} \bmod d: i \in[1, n]\right\}\right|=1$. In this case, $I=[1, q]$ works.

CASE 2: $\left|\left\{a_{i} \bmod d: i \in[1, n]\right\}\right|=2$. Suppose that

$$
\left\{a_{i} \bmod d: i \in[1, n]\right\}=\left\{r \bmod d, r^{\prime} \bmod d\right\},
$$

where $r, r^{\prime} \in[0, d-1], r \not \equiv r^{\prime}(\bmod d)$, and $a_{i} \equiv r(\bmod d)$ for at least $n / 2$ values of $i \in[1, n]$. Choose $I_{0} \subseteq\left\{i \in[1, n]: a_{i} \equiv r(\bmod d)\right\}$ with $\left|I_{0}\right|=q \leq n / 2$. Let $i_{0} \in I_{0}$ and $j_{0} \in \bar{I}_{0}=[1, n] \backslash I_{0}$ with $a_{j_{0}} \equiv r^{\prime}(\bmod d)$. When $\sum_{j \in \bar{I}_{0}} a_{j} \equiv 0(\bmod d)$, we have both

$$
\sum_{i \in\left(I_{0} \backslash\left\{i_{0}\right\}\right) \cup\left\{j_{0}\right\}} a_{i} \equiv 0-r+r^{\prime} \not \equiv 0(\bmod d)
$$

and

$$
\sum_{j \in\left(\bar{I}_{0} \backslash\left\{j_{0}\right\}\right) \cup\left\{i_{0}\right\}} a_{j} \equiv 0-r^{\prime}+r \not \equiv 0(\bmod d) .
$$

Thus, there always exists an $I \subseteq[1, n]$ with $|I|=q$ such that

$$
\left|\left\{a_{i} \bmod d: i \in I\right\}\right|=1 \quad \text { or } \sum_{i \in I} a_{i} \not \equiv 0(\bmod d),
$$

and also $\sum_{j \in \bar{I}} a_{j} \not \equiv 0(\bmod d)$.

CASE 3: $\left|\left\{a_{i} \bmod d: i \in[1, n]\right\}\right|>2$. Note that $q \geq d>2$ in this case. As $n \geq 2 q \geq 2 q-1$, by the EGZ theorem there is an $I_{0} \subseteq[1, n]$ with $\left|I_{0}\right|=q$ such that $\sum_{i \in I_{0}} a_{i} \equiv 0(\bmod q)$. For $\bar{I}_{0}=[1, n] \backslash I_{0}$, we clearly have $\left|\bar{I}_{0}\right|=(m-1) q$. Set $b=a_{1}+\cdots+a_{n} \equiv \sum_{j \in \bar{I}_{0}} a_{j}(\bmod q)$. 
Suppose that $a_{j}-a_{i} \equiv 0$ or $b(\bmod d)$ for any $i \in I_{0}$ and $j \in \bar{I}_{0}$. Then $\left|\left\{a_{i} \bmod d: i \in I_{0}\right\}\right| \leq 2$ and $\left|\left\{a_{j} \bmod d: j \in \bar{I}_{0}\right\}\right| \leq 2$.

If $i_{1}, i_{2} \in I_{0}, j \in \bar{I}_{0}$ and $a_{j} \not \equiv a_{i_{1}}, a_{i_{2}}(\bmod d)$, then $a_{j}-a_{i_{1}} \equiv b \equiv a_{j}-a_{i_{2}}$ $(\bmod d)$ and hence $a_{i_{1}} \equiv a_{i_{2}}(\bmod d)$. So, if $\left|\left\{a_{i} \bmod d: i \in I_{0}\right\}\right|=2$ then $\left\{a_{j} \bmod d: j \in \bar{I}_{0}\right\} \subseteq\left\{a_{i} \bmod d: i \in I_{0}\right\}$, which contradicts $\mid\left\{a_{i} \bmod d:\right.$ $\left.i \in I_{0}\right\} \mid>2$. Similarly, if $\left|\left\{a_{j} \bmod d: j \in \bar{I}_{0}\right\}\right|=2$ then we also have a contradiction. When

$$
\left|\left\{a_{i} \bmod d: i \in I_{0}\right\}\right|=\left|\left\{a_{j} \bmod d: j \in \bar{I}_{0}\right\}\right|=1,
$$

we cannot have $\left|\left\{a_{i} \bmod d: i \in[1, n]\right\}\right|>2$.

By the above, there are $i_{0} \in I_{0}$ and $j_{0} \in \bar{I}_{0}$ such that

Set

$$
a_{j_{0}}-a_{i_{0}} \not \equiv 0, b(\bmod d) .
$$

$$
I=\left(I_{0} \backslash\left\{i_{0}\right\}\right) \cup\left\{j_{0}\right\} \quad \text { and } \quad \bar{I}=[1, n] \backslash I=\left(\bar{I}_{0} \backslash\left\{j_{0}\right\}\right) \cup\left\{i_{0}\right\} .
$$

Then

$$
\sum_{i \in I} a_{i}=\sum_{i \in I_{0}} a_{i}-a_{i_{0}}+a_{j_{0}}=0-a_{i_{0}}+a_{j_{0}} \not \equiv 0(\bmod d)
$$

and

$$
\sum_{j \in \bar{I}} a_{j}=\sum_{j \in \bar{I}_{0}} a_{j}-a_{j_{0}}+a_{i_{0}} \equiv b+a_{i_{0}}-a_{j_{0}} \not \equiv 0(\bmod d) .
$$

Note that $|I|=q$ and $|\bar{I}|=(m-1) q$.

Combining the above and using an induction argument, we see that the desired result holds for any $m=2,3,4, \ldots$.

Lemma 2.2. Let $a_{1}, \ldots, a_{n} \in \mathbb{Z}$ with $n=p^{\alpha}$, where $p$ is an odd prime and $\alpha$ is a positive integer. If $\sum_{k=1}^{n} a_{k} \not \equiv 0(\bmod p)$ or $\mid\left\{a_{k} \bmod p: k \in\right.$ $[1, n]\} \mid=1$, then there exists a permutation $\sigma \in S_{n}$ such that $\sum_{k=1}^{n} k a_{\sigma(k)}$ $\equiv 0(\bmod n)$.

Proof. If $a:=\sum_{k=1}^{n} a_{k} \not \equiv 0(\bmod p)$, then there is an $l \in[1, n]$ such that $a l+\sum_{k=1}^{n} k a_{k} \equiv 0\left(\bmod p^{\alpha}\right)$ and hence

$$
\sum_{k=1}^{n} k a_{\sigma(k)} \equiv \sum_{k=1}^{n}(k+l) a_{k} \equiv \sum_{k=1}^{n} k a_{k}+l a \equiv 0\left(\bmod p^{\alpha}\right),
$$

where $\sigma(k)$ is the least positive residue of $k-l$ modulo $n$.

In the case $a_{1} \equiv \cdots \equiv a_{n}(\bmod p)$, it is clear that

$$
\sum_{k=1}^{p} k a_{k} \equiv a_{1} \sum_{k=1}^{p} k=a_{1} p \frac{p+1}{2} \equiv 0(\bmod p) .
$$

Thus we have the desired result for $\alpha=1$.

Now let $\alpha>1$ and assume the desired result with $\alpha$ replaced by $\alpha-1$. As mentioned above, the desired result holds if $\sum_{k=1}^{n} a_{k} \not \equiv 0(\bmod p)$. Suppose 
that $a_{1} \equiv \cdots \equiv a_{n}(\bmod p)$ and set $b_{k}=\left(a_{k}-a_{1}\right) / p$ for $k=1, \ldots, n$. In light of Lemma 2.1, there exists a partition $I_{1} \cup \cdots \cup I_{p}$ of $[1, n]$ with $\left|I_{1}\right|=\cdots=$ $\left|I_{p}\right|=p^{\alpha-1}$ such that for any $s=1, \ldots, p$ either $\left|\left\{b_{k} \bmod p: k \in I_{s}\right\}\right|=1$ or $\sum_{k \in I_{s}} b_{k} \not \equiv 0(\bmod p)$. By the induction hypothesis, there are one-to-one mappings $\sigma_{s}:\left[1, p^{\alpha-1}\right] \rightarrow I_{s}(s=1, \ldots, p)$ such that

$$
\sum_{k=1}^{p^{\alpha-1}} k b_{\sigma_{s}(k)} \equiv 0\left(\bmod p^{\alpha-1}\right) \quad \text { for all } s=1, \ldots, p .
$$

For $s \in[1, p]$ and $t \in\left[1, p^{\alpha-1}\right]$ define $\sigma\left(p^{\alpha-1}(s-1)+t\right)=\sigma_{s}(t)$. Then $\sigma \in S_{n}$ and

$$
\begin{aligned}
\sum_{k=1}^{n} k a_{\sigma(k)} & =\sum_{k=1}^{n} k a_{1}+p \sum_{k=1}^{n} k b_{\sigma(k)} \\
& =\frac{p^{\alpha}\left(p^{\alpha}+1\right)}{2} a_{1}+p \sum_{s=1}^{p} \sum_{t=1}^{p^{\alpha-1}}\left(p^{\alpha-1}(s-1)+t\right) b_{\sigma_{s}(t)} \\
& \equiv p \sum_{s=1}^{p} \sum_{t=1}^{p^{\alpha-1}} t b_{\sigma_{s}(t)} \equiv 0\left(\bmod p^{\alpha}\right)
\end{aligned}
$$

This concludes the induction step and we are done.

3. Proof of Theorem 1.2. We use induction on $\nu(n)$, the total number of prime divisors of $n$.

In the case $\nu(n)=1$, clearly $n=2$ and the desired result holds trivially.

Now let $\nu(n)>1$ and assume the desired result for those even positive integers with fewer than $\nu(n)$ prime divisors.

CASE 1: $n=2^{\alpha}$ for some $\alpha \geq 2$. By the EGZ theorem, there is an $I \subseteq[1, n]$ with $|I|=n / 2=2^{\alpha-1}$ such that $\sum_{i \in I} a_{i} \equiv 0\left(\bmod 2^{\alpha-1}\right)$. Note that for $\bar{I}=[1, n] \backslash I$ we also have

$$
\sum_{j \in \bar{I}} a_{j}=\sum_{k=1}^{n} a_{k}-\sum_{i \in I} a_{i} \equiv 0\left(\bmod 2^{\alpha-1}\right) .
$$

By the induction hypothesis, for some one-to-one mappings $\sigma_{0}:[1, n / 2] \rightarrow I$ and $\sigma_{1}:[1, n / 2] \rightarrow \bar{I}$ we have

$$
2 \sum_{k=1}^{2^{\alpha-1}} k a_{\sigma_{0}(k)} \equiv 2 \sum_{k=1}^{2^{\alpha-1}} k a_{\sigma_{1}(k)} \equiv 0\left(\bmod 2^{\alpha-1}\right) .
$$


Observe that

$$
\sum_{k=1}^{2^{\alpha-1}}(2 k-1) a_{\sigma_{1}(k)} \equiv 2 \sum_{k=1}^{2^{\alpha-1}} k a_{\sigma_{1}(k)}-\sum_{j \in \bar{I}} a_{j} \equiv 0\left(\bmod 2^{\alpha-1}\right) .
$$

For $k \in[1, n / 2]$ and $r \in[0,1]$ define $\sigma(2 k-r)=\sigma_{r}(k)$. Then $\sigma \in S_{n}$ and

$$
\sum_{j=1}^{n} j a_{\sigma(j)}=2 \sum_{k=1}^{2^{\alpha-1}} k a_{\sigma_{0}(k)}+\sum_{k=1}^{2^{\alpha-1}}(2 k-1) a_{\sigma_{1}(k)} \equiv 0\left(\bmod 2^{\alpha-1}\right) .
$$

Thus we have the desired result for $n=2^{\alpha}$.

CASE 2: $n$ has an odd prime divisor $p$. Write $n=p^{\alpha} m$ with $\alpha, m>0$ and $p \nmid m$. In view of Lemma 2.1 there is a partition $I_{1} \cup \cdots \cup I_{m}$ of $[1, n]$ with $\left|I_{1}\right|=\cdots=\left|I_{m}\right|=p^{\alpha}$ such that for each $s=1, \ldots, m$ either $\mid\left\{a_{i} \bmod p\right.$ : $\left.i \in I_{s}\right\} \mid=1$ or $\sum_{i \in I_{s}} a_{i} \not \equiv 0(\bmod p)$. Combining this with Lemma 2.2, we see that for each $s \in[1, m]$ there is a one-to-one mapping $\sigma_{s}:\left[1, p^{\alpha}\right] \rightarrow I_{s}$ such that $\sum_{t=1}^{p^{\alpha}} t a_{\sigma_{s}(t)} \equiv 0\left(\bmod p^{\alpha}\right)$.

Set $b_{s}=\sum_{k \in I_{s}} a_{k}$ for $s=1, \ldots, m$. Then

$$
\sum_{s=1}^{m} b_{s}=\sum_{k \in I_{1} \cup \cdots \cup I_{m}} a_{k}=\sum_{k=1}^{n} a_{k} \equiv 0(\bmod m) .
$$

As $2 \mid m$ and $\nu(m)<\nu(n)$, by the induction hypothesis, for some $\tau \in S_{m}$ we have

$$
2 \sum_{s=1}^{m} s b_{\tau(s)} \equiv 0(\bmod m)
$$

and hence

$$
2 \sum_{s=1}^{m} \sum_{t=1}^{p^{\alpha}} s a_{\sigma_{\tau(s)}(t)}=2 \sum_{s=1}^{m} s b_{\tau(s)} \equiv 0(\bmod m) .
$$

Note also that

$$
\sum_{s=1}^{m} \sum_{t=1}^{p^{\alpha}} t a_{\sigma_{\tau(s)}(t)}=\sum_{s=1}^{m} \sum_{t=1}^{p^{\alpha}} t a_{\sigma_{s}(t)} \equiv 0\left(\bmod p^{\alpha}\right) .
$$

Therefore

$$
2 \sum_{s=1}^{m} \sum_{t=1}^{p^{\alpha}}\left(p^{\alpha} s+m t\right) a_{\sigma_{\tau(s)}(t)} \equiv 0\left(\bmod p^{\alpha} m\right) .
$$

As $p^{\alpha}$ is relatively prime to $m$,

$$
\left\{p^{\alpha} s+m t: s \in[1, m] \text { and } t \in\left[1, p^{\alpha}\right]\right\}
$$

is a complete system of residues modulo $n=p^{\alpha} m$. For any $k \in[1, n]$, there are unique $s \in[1, m]$ and $t \in\left[1, p^{\alpha}\right]$ such that $k \equiv p^{\alpha} s+m t(\bmod n)$, and 
we define $\sigma(k)=\sigma_{\tau(s)}(t)$. Then $\sigma \in S_{n}$ and also

$$
2 \sum_{k=1}^{n} k a_{\sigma(k)} \equiv 0(\bmod n) .
$$

This concludes the induction step.

In view of the above, we have completed the proof of Theorem 1.2.

Acknowledgments. The first author is supported by Natural Science Research Project of Ordinary Universities in Jiangsu Province (grant 08KJB110002) of China and the second author is supported by the National Natural Science Foundation (grant 10871087) of China.

\section{References}

[1] A. Bialostocki, Some problems in view of recent developments of the Erdös-GinzburgZiv theorem, Integers 7 (2007), A07, 10 pp.

[2] Y. Caro, Zero-sum problems - a survey, Discrete Math. 152 (1996), 93-113.

[3] P. Erdős, A. Ginzburg and A. Ziv, Theorem in additive number theory, Bull. Res. Council Israel 10F (1961), 41-43.

[4] D. J. Grynkiewicz, A weighted Erdős-Ginzburg-Ziv theorem, Combinatorica 26 (2006), 445-453.

[5] -, On the number of m-term zero-sum subsequences, Acta Arith. 121 (2006), $275-298$.

[6] Y. O. Hamidoune, O. Ordaz and A. Ortuño, On a combinatorial theorem of Erdös, Ginzburg and Ziv, Combin. Probab. Comput. 7 (1998), 403-412.

[7] Z. W. Sun, Zero-sum problems for abelian p-groups and covers of the integers by residue classes, Israel J. Math. 170 (2009), 235-252.

[8] R. Thangadurai, Non-canonical extensions of Erdös-Ginzburg-Ziv theorem, Integers 2 (2002), A07, 14 pp.

Department of Mathematics

Huaiyin Normal University

Huaian 223300

People's Republic of China

E-mail: guosong77@hytc.edu.cn
Department of Mathematics Nanjing University Nanjing 210093 People's Republic of China E-mail: zwsun@nju.edu.cn 